\title{
RPA regulates telomerase action by providing Est1p access to chromosome ends
}

\author{
Vera Schramke $^{1,4}$, Pierre Luciano ${ }^{1,4}$, Vanessa Brevet ${ }^{2}$, Sylvine Guillot ${ }^{1}$, Yves Corda $^{1}$, Maria Pia Longhese $^{3}$, \\ Eric Gilson ${ }^{2} \&$ Vincent Géli ${ }^{1}$
}

\begin{abstract}
Replication protein A (RPA) is a highly conserved single-stranded DNA-binding protein involved in DNA replication, recombination and repair. We show here that RPA is present at the telomeres of the budding yeast Saccharomyces cerevisiae, with a maximal association in $\mathrm{S}$ phase. A truncation of the $\mathrm{N}$-terminal region of Rfa2p (associated with the $\mathrm{rfa} 2 \Delta 40 \mathrm{mutated}$ allele) results in severe telomere shortening caused by a defect in the in vivo regulation of telomerase activity. Cells carrying $r f a 2 \Delta 40$ show impaired binding of the protein Est1p, which is required for telomerase action. In addition, normal telomere length can be restored by expressing a Cdc13-Est1p hybrid protein. These findings indicate that RPA activates telomerase by loading Est1p onto telomeres during $\mathrm{S}$ phase. We propose a model of in vivo telomerase action that involves synergistic action of RPA and Cdc13p at the G-rich 3' overhang of telomeric DNA.
\end{abstract}

Replication protein A (RPA) is a eukaryotic single-stranded DNA-binding protein involved in processes including replication, recombination, transcription, checkpoints, telomere maintenance and DNA repair ${ }^{1-3}$. Human and yeast RPA are each composed of three subunits of 70, 32 and $14 \mathrm{kDa}^{4}$. RPA70 and RPA32/34 (in yeast Rfalp and $R f a 2 p$ ) become phosphorylated in response to DNA damage ${ }^{5,6}$. After DNA damage, phosphorylation of Rfalp depends on the checkpoint proteins Meclp, Mec3p, Rad9p and Rad53p whereas phosphorylation of Rfa $2 p$ depends mainly on Meclp $p^{5,6}$. Rfa2p is also phosphorylated during the normal cell cycle-in a process that depends on the yeast homologs of the related PI3-like protein kinases ataxia-telangiectasia mutated (ATM) and ATM-Rad3-related (ATR) Meclp and Tellp (ref. 5)and during meiosis ${ }^{7}$. Finally, we have shown that inactivation of the histone H3 methyltransferase Setlp induces a MEC1- and TEL1independent hyperphosphorylation of Rfa2p in G1 phase in a process that depends on the checkpoint kinase Rad53p (ref. 8). Notably, in set $1 \Delta$ cells, a truncated form of Rfa2p lacking 38 amino acids from the $\mathrm{N}$ terminus of $\mathrm{Rfa} 2 \mathrm{p}(\mathrm{Rfa} 2 \mathrm{p} \Delta 40)$, is still phosphorylated in S phase by Meclp but is not phosphorylated in G1 phase (ref. 8). The rfa2 $\Delta 40$ mutation conveys checkpoint proficiency, increased viability of checkpoint mutants subjected to irradiation, and slightly slower DNA replication (a delay of $15 \mathrm{~min}$ as compared to wild type $)^{8}$.

Telomeres are essential to the stability of chromosome ends. In most organisms, including yeast and vertebrates, telomeric DNA consists of a tandem array of short guanine-rich repeats ${ }^{9}$. The telomeric repeats are synthesized by a unique ribonucleoprotein reverse transcriptase called telomerase, which specifically extends the $3^{\prime}$ G-rich telomeric strand ${ }^{10}$. Synthesis of the complementary $5^{\prime}$ C-rich telomeric strand is thought to be driven by the DNA polymerase $\alpha$-primase complex ${ }^{11}$. In S. cerevisiae, the $3^{\prime}$ ends of telomeric DNA consist of a G-rich single-stranded stretch that is detected only during $S$ phase ${ }^{12}$. This G-tail associates with two singlestranded DNA-binding proteins, Cdc13p and Estlp, that are required for telomerase activity in vivo ${ }^{13-15}$. The action of telomerase at the telomere itself is cell-cycle restricted, indicating that a switch in telomere structure may be required for telomerase action $^{16-19}$. This process is related to the interaction of the telomerase catalytic subunit (Est2p) with Cdc13p and Est1p (refs. $14,15,18)$. Est $2 p$ seems to be present at telomeres throughout the cell cycle, in contrast to Estlp, which binds telomeres mainly in $S$ phase in a fashion that seems to be preserved in the presence of the defective $c d c 13-2$ allele $^{18}$. It has therefore been proposed that Est1p binds telomeres in late $S$ phase and interacts with Cdc13p to activate telomere-bound Est2p (ref. 18).

Here we investigated the relationships between RPA and telomeres. We show that RPA is required to maintain telomere length and acts in a pathway that involves Est $2 p$ and the telomerase positive regulator Tel1p. Our results indicate that RPA regulates the action of telomerase during the cell cycle by specifically facilitating the binding of Est1p to telomeres during $\mathrm{S}$ phase.

\footnotetext{
${ }^{1}$ Laboratoire d'Ingénierie des Systèmes Macromoléculaires, Centre Nationale de la Recherche Scientifique (CNRS), 31 chemin Joseph Aiguier, 13402 Marseille Cedex 20, France. '2Laboratoire de Biologie Moléculaire de la Cellule, CNRS, Unité Mixte de Recherche 5161, Ecole Normale Supérieure de Lyon, 46 allée d'Italie, 69364 Lyon Cedex, France. ${ }^{3}$ Dipartimento di Biotecnologie e Bioscienze, Università di Milano-Bicocca, Piazza della Scienza 2,20126 Milano, Italy. ${ }^{4}$ These authors contributed equally to this work. Correspondence should be addressed to V.G. (e-mail: geli@ibsm.cnrs-mrs.fr).
} 


\section{RESULTS}

\section{Binding of Rfa2p to telomeric DNA}

We investigated whether Rfa2p is bound in vivo to telomeric DNA. We first used a one-hybrid assay for telomere-binding proteins ${ }^{20}$ in which Rfa2p was fused to a transcriptional activation domain and tested for its ability to activate transcription of a telomere-linked, promoter-defective HIS 3 allele. We compared transcriptional activation of the telomeric HIS3 reporter by the Rfa2p fusion protein to transcriptional activation by similar fusion proteins of Rif2p (Rif2p binds telomeres through its interaction with the telomeric DNA binding protein Raplp) and of Cdc13N (the N-terminal domain of Cdc13p, which does not contain the DNA-binding domain). The Rfa2p fusion protein induced transcriptional activation of the telomere-linked HIS3, indicating that Rfa2p is a telomere-binding protein (Fig. 1a). The Rfa2p fusion protein produced weaker transcriptional activation than the Rif2 $p$ fusion protein but stronger activation than the Cdc13N fusion protein. Unlike Rif2p, Rfa2p did not activate the HIS3 reporter gene when it was adjacent to an internal tract of telomeric sequences (Fig. 1a). This indicates that in vivo binding of Rfa2p may require the presence of a chromosome end.
To confirm the association of Rfa2p with telomeric DNA, we carried out chromatin immunoprecipitation (ChIP) assays. We used cells carrying the URA3 reporter inserted next to the left telomere of chromosome VII and expressing a Myc-tagged version of Rfa2p (UCC1001 RFA2-MYC). We considered the Rfa2-Myc fusion protein to be functional: the RFA2-MYC cells behaved like wild-type cells with respect to growth, DNA replication, biological responses to DNA damage and telomeric position effects, although their telomeres were slightly ( $30 \mathrm{bp}$ ) longer than those of wild-type cells (data not shown). Chromatin from RFA2-MYC cells was crosslinked, immunoprecipitated and analyzed by PCR. Rfa2-Myc immunoprecipitates allowed amplification of the telomeric PCR products pUra1 and pUra2, or pTelVI, but not of the control pBdf1 PCR fragment (Fig. 1b). When cells without the Myc-tagged Rfa2p were subjected to the same ChIP assay, or when immunoprecipitation was done in the absence of in vivo formaldehyde cross-linking, we did not detect any PCR amplification (Fig. 1b), demonstrating the specificity of the ChIP.

Next we addressed whether Rfa2p showed a cell cycle-regulated association with telomeres. We released cells expressing the chromosomally a

Figure 1 Rfa2p binds to telomeric DNA in vivo. (a) Rfa2p fusion protein activates HIS3 at a telomere but not at an internal tract of telomeric DNA. Proteins fused to the B42 activation domain were expressed in strains (YM701 background) in which the HIS3 promoter-defective allele was inserted, either adjacent to an internal tract of telomeric DNA (HIS-Int-CA) or immediately adjacent to the left telomere of chromosome VII (HISTel) ${ }^{20}$. Cells were spotted onto test plates (-His+3AT) or control plates (-Trp). (b) Scheme showing the fragmented URA3 gene at the left arm of chromosome VII (left), the telomere at the right arm of chromosome VI (ChR-VI; right) and BDF1 at chromosome XII (top).

Primer pairs used to amplify telomeric fragments pUra1 and pUra2, Chr-VI-R telomere fragment pTeIVI and control nontelomeric fragment pBdf1 are indicated. The ChIP assay was carried out with antibodies to Myc (9E10) on chromatin from the indicated UCC1001 strains. After DNA purification, PCR was carried out with telomeric primers ( $\mathrm{pUra} 1, \mathrm{pUra} 2$ and pTelVI) and nontelomeric control primers (pBdf1). (c) Association of Rfa2p with telomeric DNA increases in $S$ phase. Right, FACS analysis of the synchronized UCC 1001 cells after release from $\alpha$-factorinduced G1 block. Left, results of ChIP assays on Myc-tagged Rfa2p, carried out on chromatin from cells harvested in exponentially growing (exp), G1 phase, $S$ phase and G2 phase cells after release from an $\alpha$-factor block. Relative enrichment of Rfa2-Myc-bound telomeric DNA (pUra2) over background ( $p B d f 1)$ was calculated as indicated in Methods. The relative amount of telomeric DNA in the precipitates of exponentially growing cells was arbitrarily taken as 1 .

b
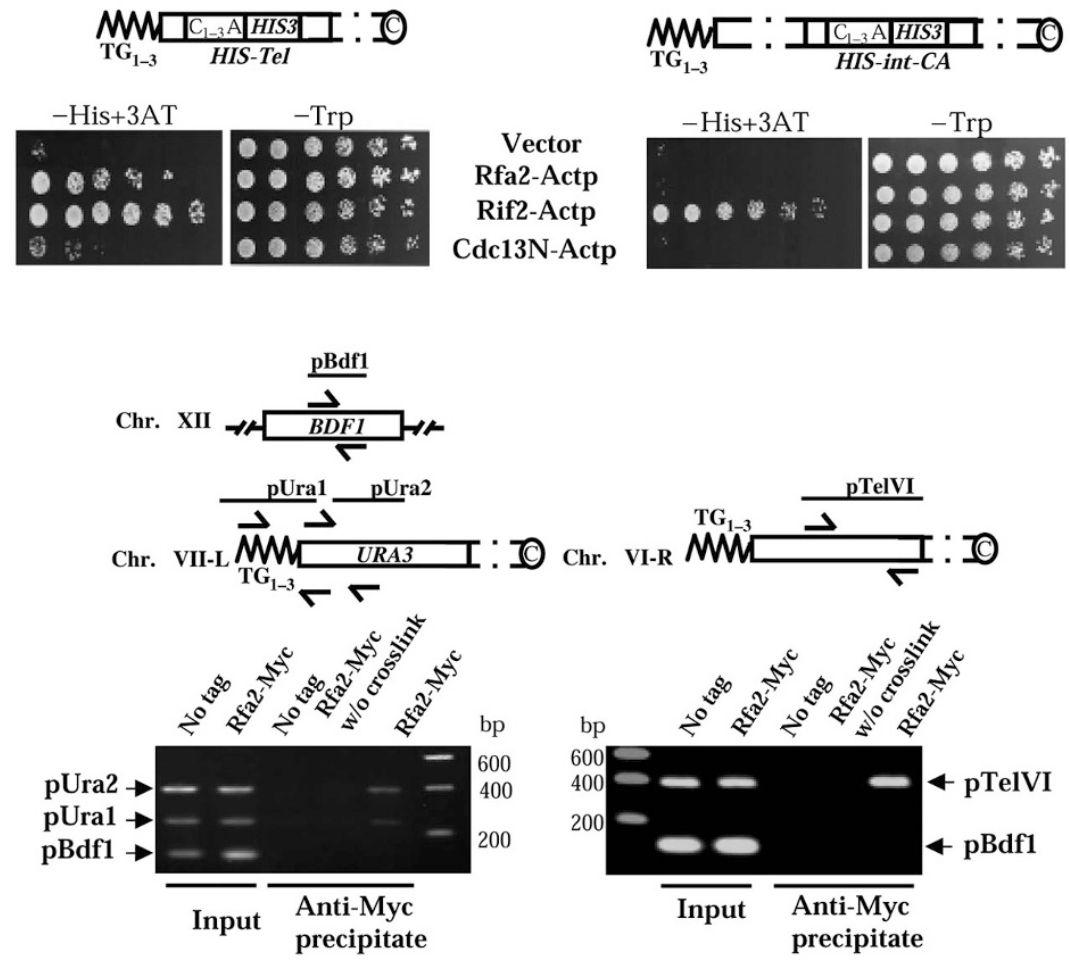

C
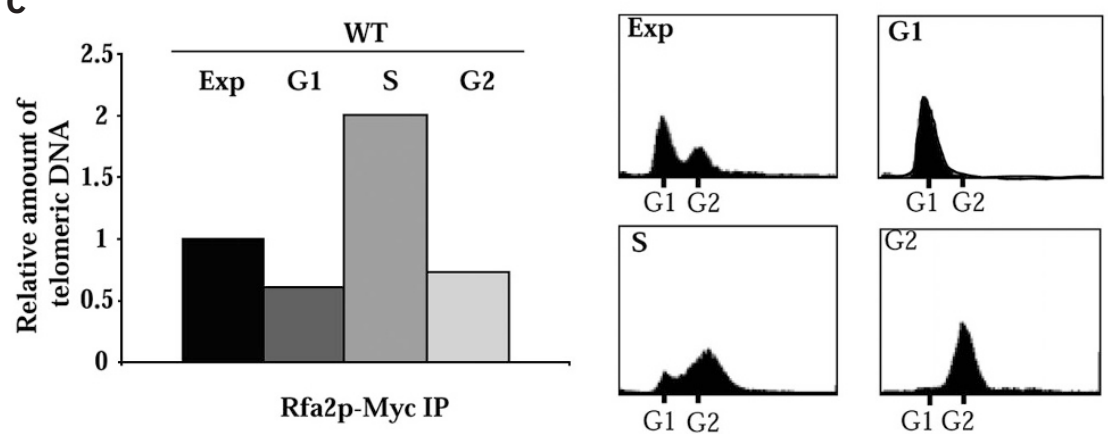
Figure 2 Rfa2p is involved in telomere length regulation. (a) Comparison of the telomere length of haploid strains isogenic to UC1001 grown for more than 100 generations carrying a subtelomeric URA3 reporter gene on the left arm of chromosome VII. Median telomere length was calculated from two independent experiments using ImageQuant (Molecular Dynamics). (b) The rfa2 440 mutation does not lead to an increase in single-stranded telomeric G-rich DNA. Genomic DNAs isolated from the indicated strains were digested with Xhol and analyzed by nondenaturing hybridization (native) followed by denaturation of the DNA (denatured) in the same gel and rehybridization with the same telomeric C1-3A/TG1-3 probe as described ${ }^{21}$. nt, nucleotide; ss, single-stranded; WT, wild type. a

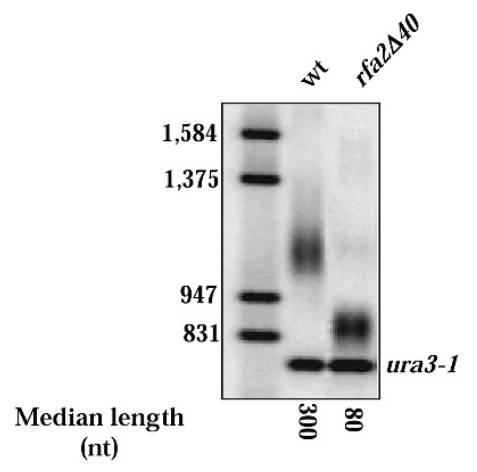

b

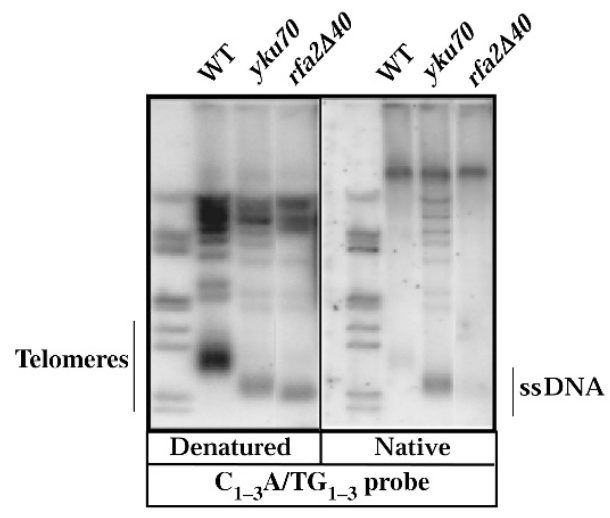

encoded Rfa2-Myc18 from an $\alpha$-factor-induced G1 block and monitored their progression through the cell cycle by FACS analysis (Fig. 1c). Cells in G1, S and G2 phases were subjected to ChIP (Fig. 1c). Amounts of DNA in the input samples and immunoprecipitates were measured by real-time PCR and normalized to the level of the nontelomeric pBdf1 fragment presents in the immunoprecipitates. PCR quantification analysis indicated that the amount of telomeric DNA bound to Rfa2p in cells harvested in S phase was four times higher than in cells harvested in G1 phase (Fig. 1c). Data showing enrichment at telomeres, as compared to a nontelomeric control of Rfalp and Rfa2p during the cell cycle, is described below.

\section{$\mathrm{Rfa} 2 \mathrm{p}$ is involved in regulation of telomere length}

We had previously constructed a mutant strain with a chromosomally encoded, truncated form of Rfa2p lacking 38 amino acids from the $\mathrm{N}$ terminus ${ }^{8}$. We introduced the mutated allele, $r f a 2 \Delta 40$, into yeast strain UCC1001, which carries a subtelomeric URA3 reporter gene, and examined the median length of the left telomere of the fragmented chromosome VII-L. Telomeres in the rfa2 $\Delta 40$ strain were critically short (220 bp shorter than wild-type telomeres; Fig. 2a). We next asked whether this telomere shortening was accompanied by an increase in the amount of single-stranded G-strands in exponentially growing cells, as is the case for cells deficient for $\mathrm{Ku}(y k u)^{21}$, a DNAbinding heterodimer that is crucial in DNA end protection. In wildtype cells, telomeric single-stranded extensions cannot be detected with telomeric probes in the native DNA of cells outside $S$ phase ${ }^{12}$. This contrasts with $y k u$ mutants, which show long terminal extensions of the G-rich strand. We did not detect long G-tails in the rfa2 $\Delta 40$ cells, although we detected them in $y k u 70$ mutants. Natural telomeres were also shortened in $r f a 2 \Delta 40$ cells (Fig. 2b).

\section{RPA acts in the telomerase pathway}

The length of the single-stranded overhang has been proposed to correlate with the rate of telomeric DNA degradation ${ }^{22}$; thus, the absence of an expanded $3^{\prime}$ overhang in $r f a 2 \Delta 40$ suggested that this allele does not affect the rate of telomeric DNA degradation. To address this prediction directly, we compared telomere length dynamics in Rfa2p and Est2p mutants. We constructed a heterozygous diploid strain (JKM179 background) carrying the rfa2 $\triangle 40 \mathrm{~N}$ terminal truncation allele and a deletion allele of EST2, est $2 \Delta$. The diploid strain RFA2/rfa2 440 ; EST2/est2 $\Delta$ :NAT, ADH4/TELadh4: $U R A 3$ was sporulated, and the growth reduction with increasing a

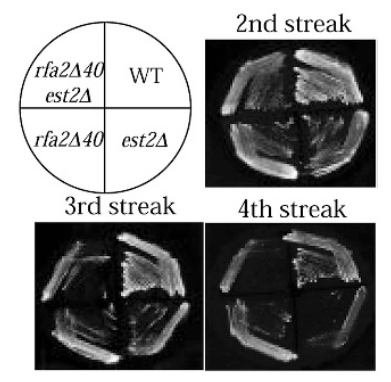

b

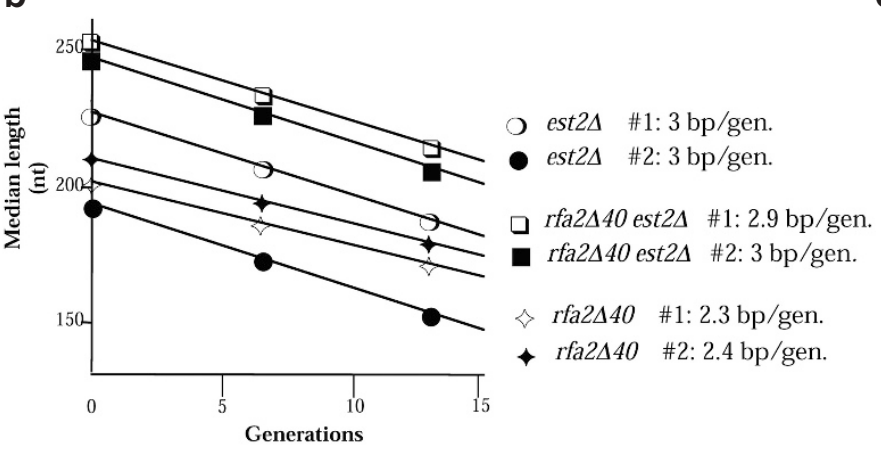

C

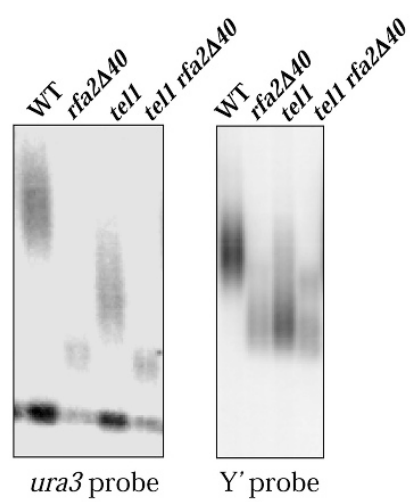

Figure 3 RFA2 acts in the telomerase pathway. (a) Viability of the est2 $\Delta$, rfa2 $\Delta 40$ and est $2 \Delta$ rfa2 $\Delta 40$ strains with increasing number of generations (gen.). Shown are successive streak cultures on YPD medium of spores generated from a single tetrad. (b) Telomere degradation rates. Telomere length of the indicated yeast strains (JKM179 background) were plotted against generation and telomeric degradation rates were calculated from linear regression curves. (c) Deleting TEL 1 does not aggravate telomere shortening of the rfa2 $\triangle 40$ mutant. Telomere length of the indicated spores was measured after 3 streakings. Left, the URA3 genetically marked telomere was analyzed as in Figure 2a. Right, lengths of $Y^{\prime}$ telomeres. Telomere length was measured by reference to molecular weight markers (SmartLadder, Eurogentec). 
generations and the rate of telomere shortening at the adh4:URA3 telomeres were measured in cell cultures established from different spores. The est $2 \Delta / r f a 2 \Delta 40$ double mutant showed a growth reduction over generations comparable to that of the est $2 \Delta$ single mutant, whereas growth reduction of the single $r f a 2 \Delta 40$ mutant was less severe (Fig. 3a). We did not notice clear signs of senescence in $r f a 2 \Delta 40$ cells, which can grow indefinitely, although at a low growth rate. In agreement with this observation, the short mean length seemed stable in long-term culture. However, after several streakings we noticed a high frequency of $r f a 2 \Delta 40$ cells with recombined telomeres and higher growth rates. Thus, telomeres were not fully stable in $r f a 2 \Delta 40$ cells.

We next quantified the rate of telomere shortening in growing cells harboring the same three mutation combinations. As expected, in cultures from wild-type spores, telomere length remained constant (data not shown); in cultures from spores lacking EST2, median telomere length decreased linearly at a rate of roughly 3 bp per generation (Fig. 3b). Notably, rates of telomere shortening were identical in cultures from est $2 \Delta$ spores with and without the rfa2 $\Delta 40$ allele (Fig. 3b). Variation in initial telomere length between individual spore-derived cultures is likely to reflect differences in telomere length in the initial diploids or differences in the number of generations since the initial diploids. Initial length is not expected to influence the degradation rate ${ }^{16}$. We concluded that the $r f a 2 \Delta 40$ allele does not increase the rate of telomeric DNA degradation resulting from the loss of telomerase. Indeed, the rate of telomere shortening in the $r f a 2 \Delta 40$ single mutant was $2.3-2.4$ bp per generation (Fig. $3 \mathbf{b}$ ), substantially lower than the $3 \mathrm{bp}$ per generation in cells lacking
EST2. This, together with the fact that $r f a 2 \Delta 40$ cells show a reduction of the median telomere length rather than a total loss of telomeric DNA, indicates that telomerase action in the $r f a 2 \Delta 40$ mutant is reduced but not abolished. Overall, we conclude that RFA2 is important for telomerase action.

The results described above prompted us to analyze the genetic interactions between RFA2 and the ATM homolog TEL1, which has been proposed to regulate telomerase activity ${ }^{23}$. We first sporulated and dissected a diploid RFA2/rfa2 440 ; TEL1/tel1; ADH4/TELadh4: URA3 strain. After two streakings starting from a spore and an overnight culture, we compared telomere lengths in isogenic tel1/rfa2 $\Delta 40$, tell and $r f a 2 \Delta 40$ mutants for both the genetically marked URA3 telomeres and telomeres carrying a conserved XhoI site in their Y' element (Fig. 3c). Although the sizes of the natural telomeres were similar in rfa2 $\Delta 40$ and tell mutants, the URA3-marked telomeres were reproducibly shorter in $r f a 2 \Delta 40$ cells than in tell cells (by about $110 \mathrm{bp}$ ). Such differences between Y' telomeres and genetically marked telomeres have been reported elsewhere ${ }^{24}$. Most notably, the genetically marked and natural telomeres in $r f a 2 \Delta 40$ and $r f a 2 \Delta 40$ tell cells were almost identical in size ( $\approx 10$ bp difference; Fig. $3 c$ c). We concluded that deleting TEL1 does not exacerbate the telomere shortening in $r f a 2 \Delta 40$ mutant cells.

\section{Telomeric binding of RPA in wild-type and $r f a 2 \Delta 40$ cells}

We investigated whether the binding of Rfa1p and Rfa2p to telomeres was altered in $r f a 2 \triangle 40$ cells. We carried out ChIP experiments at different stages of the cell cycle in RFA1-MYC and RFA1-MYC/rfa2 $\triangle 40$ cells, using polyclonal antibodies to Rfa2 and monoclonal antibodies to Myc

a

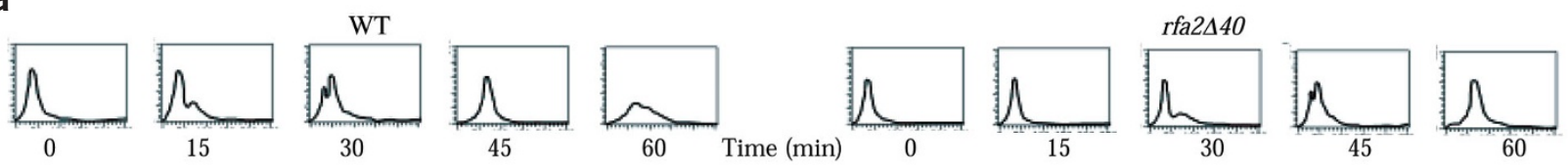

b
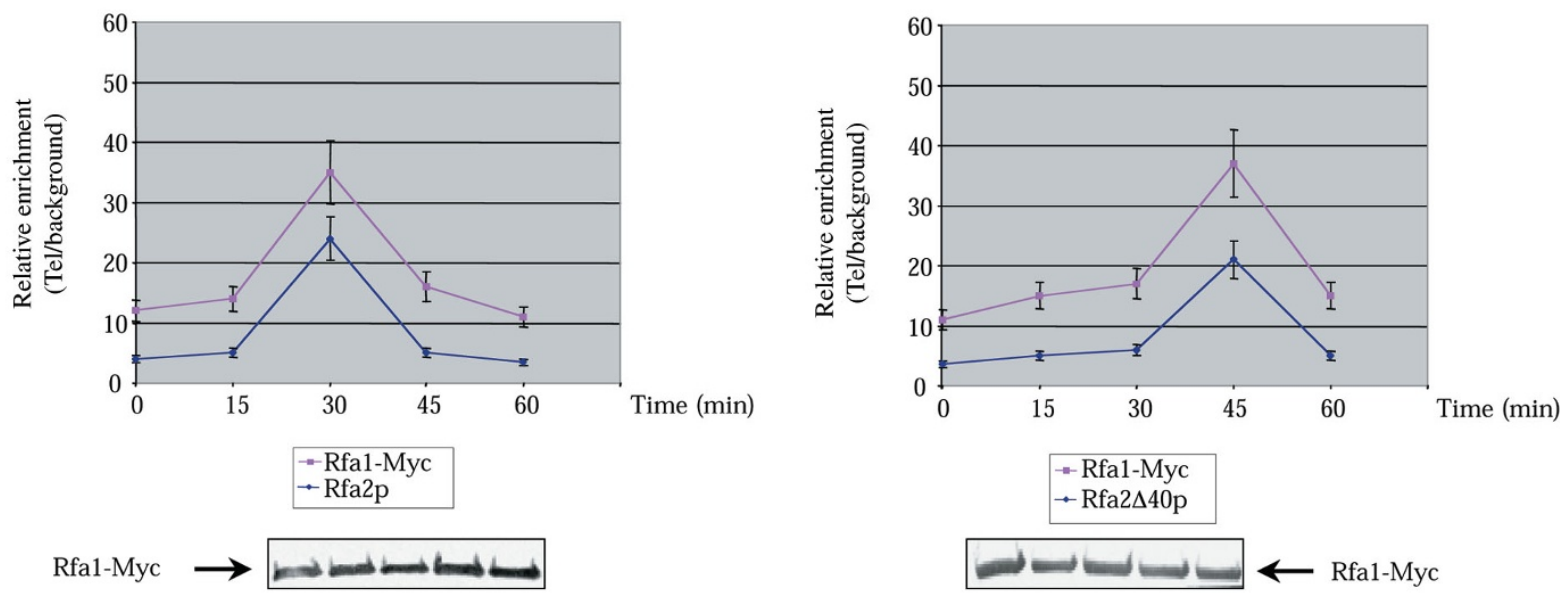

$\mathrm{Rfa} 2 \mathrm{p}$
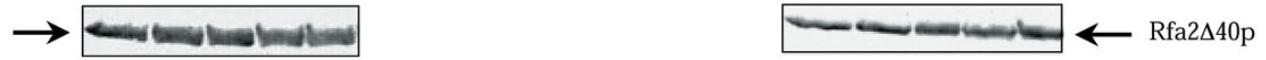

Figure 4 Telomeric association of Rfa2p (or Rfa2 $440 p$ ) and Rfa1p in wild-type (WT) and rfa2 440 cells. (a) FACS analysis of the synchronized UC1001 cells after release from $\alpha$-factor-induced G1 block. (b) Amounts of telomeric DNA (pUra2 or Tel) determined by ChIP assays of chromatin from cells harvested every $15 \mathrm{~min}$ after release from an $\alpha$-factor block. The amount of DNA was calculated by quantitative real-time PCR. Relative enrichment of Rfa2p- and Rfa1-Myc-bound telomeric DNA (pUra2/Tel) over background (pBdf1) is plotted against time. Amounts of Rfa2p, Rfa2 $\Delta 40 p$ or Rfalp-Myc immunoprecipitated protein at each time point are shown. 
to monitor the binding of Rfa2p and a functional Rfa1-Myc fusion, respectively (Fig. 4). In the course of the cell cycle, the truncated version of Rfa2p bound to telomeres much like full-length Rfa2p. Like $\mathrm{Rfa} 2 \mathrm{p}, \mathrm{Rfa} 1 \mathrm{p}$ bound to telomeres with a maximum in late $\mathrm{S}$ phase, and this was true in the rfa2 $\Delta 40$ strain as well as in the wild type (Fig. 4). Thus, $r f a 2 \Delta 40$ does not alter RPA binding to telomeres. We also found that Rfa2p was coimmunoprecipitated with Rfa1p-Myc and vice versa, and that Rfa2 $\Delta 40 \mathrm{p}$ was also coimmunoprecipitated with Rfalp-Myc and vice versa (data not shown). We therefore concluded that Rfalp and Rfa $2 p$ both bind to telomeres with similar kinetics and that truncating the $\mathrm{N}$ terminus of Rfa2p does not affect the telomeric binding of $\mathrm{Rfa} 2 \mathrm{p}$ and Rfa1p. Consistent with these results, Rfa2 $\Delta 40 \mathrm{p}$ still interacts with Rfalp. Taken together, these data indicate that the role of RPA in telomere maintenance is most likely to involve the whole RPA heterotrimer. It is likely that an activity of RPA other than DNA-binding activity is responsible for the telomeric phenotype shown by rfa2 $\Delta 40$ mutant cells.

Binding levels of Rfa1-Myc (anti-Myc ChIP) and Rfa2p (antiRfa2p ChIP) to telomeric DNA in G1 phase was five and ten times, respectively, that for the nontelomeric genomic control (Fig. 4). By comparison, under the same ChIP conditions, telomeric binding of Myc-tagged Est1p, Cdc13p and Est2p in G1 phase was 4, 4.6 and 36 times, respectively, that of the control (Fig. 5). We therefore believe that the amount of RPA bound to telomeres in G1 phase is functionally important though low. The difference in the telomeric binding of Rfa2p and Rfa1-Myc may reflect a slightly higher background binding when antibodies to Rfa2p, as compared to antibodies to Myc, are used.

\section{$\mathrm{Rfa} 2 \mathrm{p}$ is required for telomeric binding of Est1p during $\mathrm{S}$ phase}

We next assessed, using ChIP assays, whether the binding of Cdc13p, Est1p and Est2p to telomeric DNA is impaired in rfa2 $\Delta 40$ cells. A Myctagged version of CDC13, EST1 or EST2 was introduced into wild-type and $r f a 2 \Delta 40$ strains bearing the URA3-marked telomere. Overall, the tagged proteins were functional. The tagged strains were synchronized in G1 phase with $\alpha$-factor and released into the cell cycle. We monitored cell-cycle progression of both the wild-type and $r f a 2 \Delta 40$ strains by FACS analysis, collecting samples every $15 \mathrm{~min}$ for ChIP assays. The amounts of telomeric DNA bound to Cdc13p and Est2p did not differ notably between the strains (Fig. 5). In both strains, telomeric binding of Cdc13p and Est2p increased in S phase (Fig. 5), although a proportion of Est2p is already associated with the telomeres in G1 phase (ref. 18). By contrast, the increase in telomeric binding of Estlp that normally occurs in $S$ phase was severely impaired in the $r f a 2 \Delta 40$ cells (Fig. 5). We concluded from these data that the $r f a 2 \Delta 40$ mutation results in a specific defect in Est1-Myc telomeric binding in S phase.

To assess whether the Est1p telomeric binding defect observed in rfa $2 \Delta 40$ cells would not simply result from a shortened or perturbed a
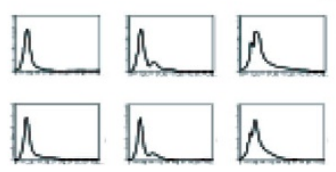

WT
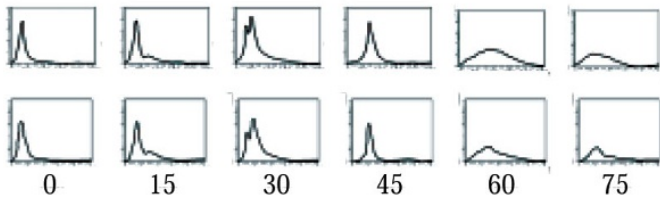

b
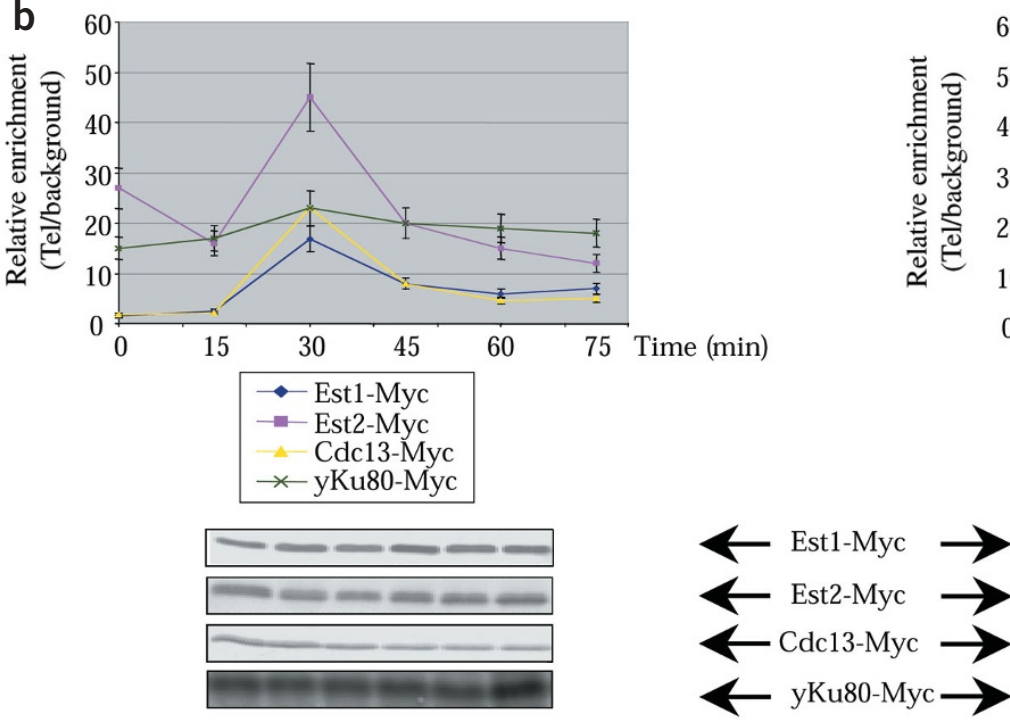

$\operatorname{rfa} 2 \Delta 40$
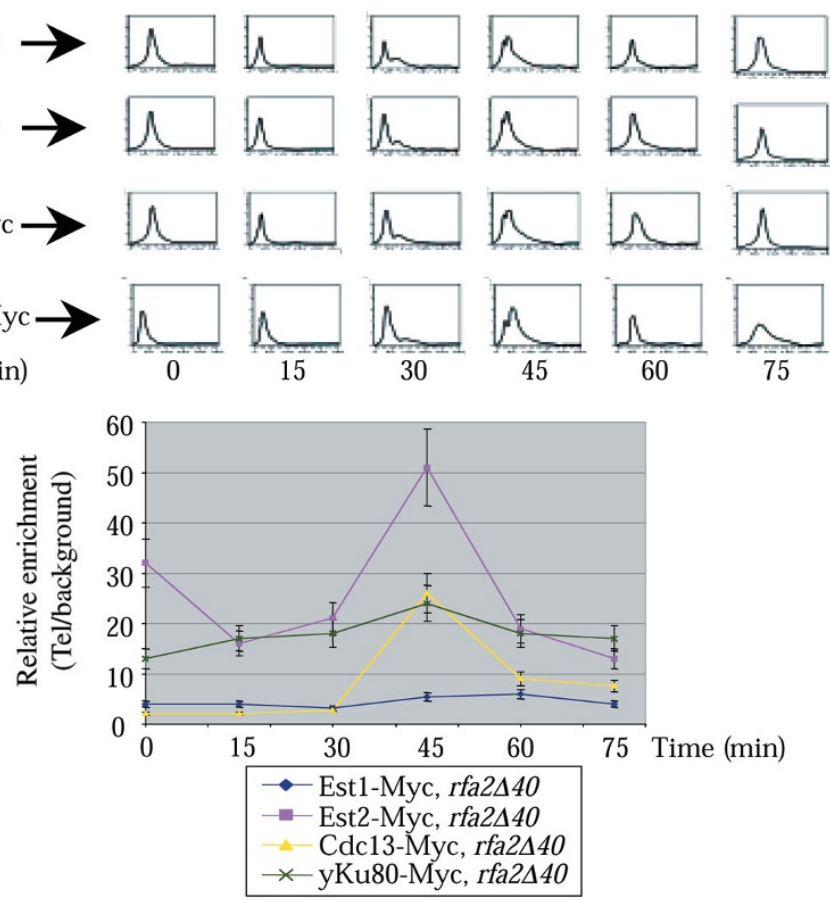

Figure 5 The rfa2 $\triangle 40$ allele impairs Est1p telomeric DNA binding during S phase. (a) FACS analysis of the indicated UC1001 cells after release from $\alpha$ factor-induced G1 block. (b) Telomeric DNA binding of Est1-myc, Est2-myc, Cdc13-myc and yKu80-myc was monitored by ChIP assays (anti-Myc (9E10)) as described in Figure 4. Relative enrichment of Est1-myc, Est2-myc, Cdc13-myc and yKu80-myc-bound telomeric DNA over background is plotted against time. Immunoprecipitated proteins at each time point were analyzed by western blotting with anti-Myc (9E10). 
telomere, we analyzed the telomeric binding of yKu $80 \mathrm{p}$ by the same method using a yKu80-Myc fusion. A perturbation of yKu at the telomere might be predicted to lead to a loss of Est1p recruitment ${ }^{25}$. Similar yKu80p binding occurred in the wild type and the $r f a 2 \Delta 40$ mutant. In both strains, yKu80p bound to telomeres throughout the cell cycle, with a slight increase in S phase (Fig. 5). Thus, the defect in Estlp recruitment in $r f a 2 \Delta 40$ cells does not seem to be related to a perturbation of yKu80p localization to the telomere. This additive control supports a direct role of RPA in Est1p access to telomeres.

Following the same logic, we next analyzed whether the binding of Cdc13p, Est1p and Est2p would be affected in a $y k u 70 \Delta$ mutant that has short telomeres (Fig. 6). We observed a slight decrease in the binding of Est2p to mutant telomeres in G1 and in $S$ phase in the $y k u 70 \Delta$ mutant, whereas binding of Cdc13p to telomeres in $\mathrm{S}$ phase was increased. Binding of Estlp to telomeres in $\mathrm{S}$ phase is slightly decreased in the $y k u 70 \Delta$ mutant, whereas binding of Est1p to telomeres in $S$ phase is severely decreased in the $r f a 2 \Delta 40$ mutant (compare Fig. 5b, wild-type and rfa $2 \Delta 40$, with Fig. 6b). From these results, we believe that the decrease of Est 1 p binding to telomeres is not an indirect consequence of telomere shortening but rather results from a direct role of RPA in enabling access of Est1p to telomeres.

\section{$r f a 2 \Delta 40$ telomere length when Est1p is tethered to the telomere}

That $r f a 2 \Delta 40$ cells show a strong defect in the telomerase pathway of telomere length regulation and an altered binding of Estlp to telomeres indicates that RPA may facilitate loading of Estlp to promote telomerase action at telomeres. If this notion is correct, one can anticipate that the $r f a 2 \Delta 40$ mutation would be suppressed if telomeric binding of Estlp were forced by expression of a hybrid protein consisting of Estlp and the DNAbinding domain of Cdc13p. We transformed wild-type and $r f a 2 \Delta 40$ cells with the plasmid pVL1120, which directs the expression of such a hybrid protein, Est1p-DBD ${ }_{\mathrm{CDC} 13}$ (ref. 14). Efficient telomere lengthening occurred in the rfa2 $\Delta 40$ mutant (>200 bp) (Fig. 7), and the growth defect of $r f a 2 \Delta 40$ cells was suppressed by the transformation with pVL1120 (data not shown). This occurred for every clone we analyzed, although the extent of telomere elongation varied between transformants. These results confirmed that $\mathrm{Cdc} 13 \mathrm{p}$ retains the ability to bind to telomeric DNA in rfa $2 \Delta 40$ mutants (as discussed above) and that the telomere length defect associated with the rfa2 $\Delta 40$ mutation can be corrected by forcing the loading of Est1p onto the telomere.

\section{DISCUSSION}

We have shown that RPA is present at telomeres and that truncation of the N-terminal region of $\mathrm{Rfa} 2 \mathrm{p}$ greatly reduces telomere length. Because RPA participates in most aspects of DNA metabolism as a singlestranded DNA-binding protein, binding of RPA to telomeres might seem to be related to a

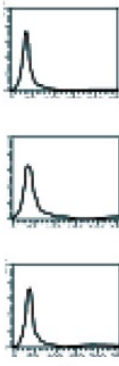

15

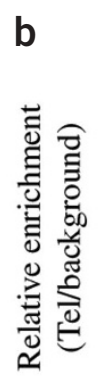

the presence of single-stranded telomeric DNA (G-tail). Consistent with this hypothesis, during the $S$ phase of the cell cycle there is a general coincidence between the maximal telomeric binding of RPA described here, G-tail formation and telomere elongation. Based on all these results, we propose that RPA facilitates telomerase action during $S$ phase.

Our ChIP analysis showed no notable differences between wildtype and $r f a 2 \Delta 40$ cells in the amount of Cdc13p and Est2p associated with telomeres. In contrast, binding of Estlp in $\mathrm{S}$ phase is severely impaired in $r f a 2 \Delta 40$ cells. Although the question was not directly addressed in this study, it is likely that $\mathrm{Cdc} 13 \mathrm{p}$ is also required for proper Estlp loading during telomerase activation ${ }^{14,15,18}$. This suggests that telomere shortening in the $r f a 2 \Delta 40$ mutant is not due to reduced binding of Cdc13p and Est $2 p$ to telomeric DNA, but rather reflects a decrease in the binding of Estlp to telomeres. This theory is in agreement with the fact that artificial tethering of Estlp to telomeres (via a Estlp-DBD $\mathrm{CDC} 13_{3}$ fusion protein) corrected the $r f a 2 \Delta 40$ associated defects. The binding of Est $2 p$ to telomeres seems to be independent of proper loading of Est1p. This is consistent with the idea that the role of Est1p is not to recruit Est2p to telomeres but
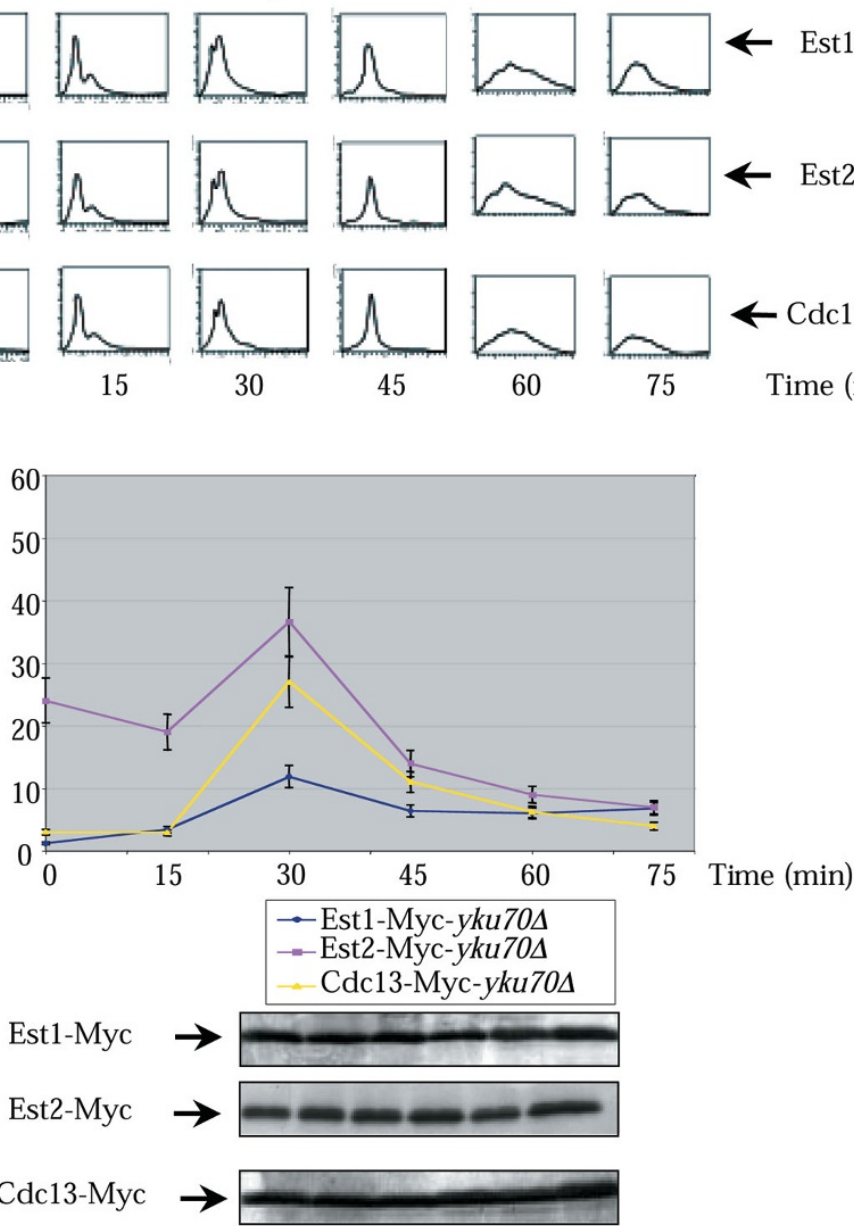

Figure 6 Association of Est1-myc, Est2-myc and Cdc13-myc in yKu70 $\Delta$ cells. (a) FACS analysis of the indicated UC1001 cells after release from $\alpha$-factor-induced G1 block. (b) Telomeric DNA binding of Est1-myc, Est2-myc and Cdc13-myc was monitored by ChIP assays as described in Figure $\mathbf{5}$. The wild-type control experiment is illustrated in Figure $\mathbf{5 b}$. 


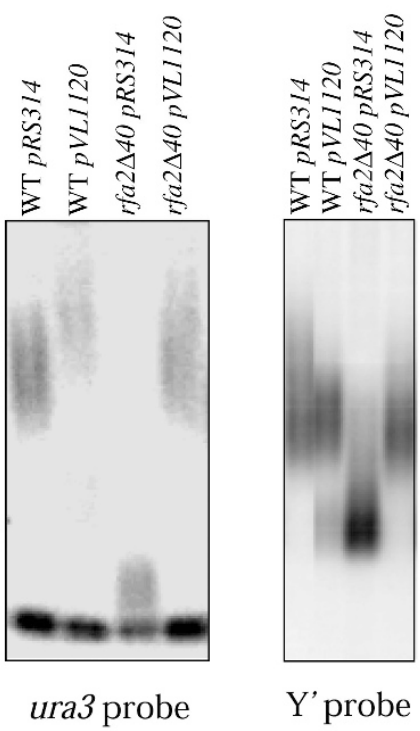

Figure 7 Telomere lengthening by targeted Est1p in rfa2 $\Delta 40$ cells. Telomere length was measured by Southern blotting using either ura3 (left) or Y' probe (right). pVL1120, which directs the expression of Est1-DBDCDC13 (ref. 14), derives from pRS314. Telomere length was measured by reference to molecular weight markers (SmartLadder, Eurogentec). WT, wild type.

rather to activate an already bound form of telomerase ${ }^{18}$. We have been unable to coimmunoprecipitate Est1p (or Cdc13p or Est2p) with Rfa2p (data not shown), indicating that control of telomerase activity by Rfa $2 p$ may not be mediated by a stable interaction between Rfa2p and Estlp. Either the putative interaction between RPA and Est $1 p$ is too infrequent, too transient or both to be detected, or RPA does not directly recruit Est1p but rather maintains singlestranded DNA in a state amenable to Estlp binding. For instance, RPA could act through the stimulation of a helicase activity, opening up the duplex portion of the telomeric DNA and thereby facilitating the binding of Estlp. Overall, we propose that RPA and Cdc13p cooperate to allow Est1p binding to telomeres and subsequently promote telomerase action (Fig. 8). The $r f a 2 \Delta 40$ allele seems to be specifically impaired in the Est1p loading step (Fig. 8).

Our results do not rule out roles for RPA in other aspects of telomere length regulation, including the control of telomeric DNA degradation and the coupling of telomerase activity to laggingstrand synthesis ${ }^{17,26}$. RPA has consistently been shown to interact with the DNA polymerase $\alpha$-primase complex ${ }^{27}$. In addition, we found that the rfa1-M2 allele ${ }^{28}$ of the large subunit of RPA (RFA1) results in telomere elongation (data not shown), indicating a possible role of RPA in $\mathrm{C}_{1-3} \mathrm{~A}$ strand synthesis by DNA polymerase $\alpha$.

We have found that deleting TEL1 in rfa2 $\triangle 40$ cells does not aggravate telomere shortening, indicating that RPA and Tellp may act in the same pathway. Notably, the phenotypes of $r f a 2 \Delta 40$ cells show similarities with the telomeric phenotypes of cells devoid of Meclp and Tellp: (i) rfa2 $\Delta 40$ cells and cells lacking Tellp and Meclp have residual telomerase activity (as described here and in refs. 29,30); (ii) the association of Cdc13p with telomeres occurs efficiently in tel1 $\Delta$ cells and $r f a 2 \Delta 40$ cells (described here and in ref. 31 ); and (iii) forcing the Cdc13p-dependent recruitment of telomerase to the telomeres suppresses the growth defects of both $r f a 2 \Delta 40$ and mecl tell cells (described here and in ref. 31). Another link between Rfa2p, Mec1p and Tell $\mathrm{p}$ is provided by the fact that Rfa2p is phosphorylated during $S$ phase mainly by Meclp but also by Tellp (ref. 5). We can speculate from these data that RPA could be one of the targets of these kinases at telomeres, although a very recent report indicates that substituting alanine residues for the two serines (Ser122 and Ser238) of Rfa2p that are in SQ motifs (preferred targets of the ATM kinase family) does not affect telomere length ${ }^{32}$. Notably, the same study showed that the rfa2-55 allele (encoding E90A and D91A substitutions produces short telomeres ${ }^{32,33}$.

The control of telomerase activity by two single-stranded DNA-binding proteins, Cdc13p and RPA, may provide a means to couple telomerase activity tightly to the formation of 3 ' overhangs during telomere replication. Thus, RPA might be a crucial link between telomere homeostasis and the replication of chromosome ends. The role of RPA in the loading of Estlp at telomeres may be evolutionarily conserved, as both RPA and Estlp are present in human as well as yeast cells ${ }^{2,34,35}$.

\section{METHODS}

Mutant strain construction and telomere experiments. A deletion removing the RFA2 intron and the region extending from codon 3 to codon 40 of RFA2 (rfa2 $\triangle 40)$ was introduced as described ${ }^{8}$. By replacing the chromosomally encoded RFA2 with the mutated rfa2 $\triangle 40 \mathrm{t}$ allele, we generated an allele ( $r f a 2-$ 165) encoding a truncated protein extending from residue 1 to residue 165 that is coexpressed with Rfa2 $\mathrm{p} \Delta 40$. To get rid of potential effects of $r f a 2-165$, this inviable, truncated allele was fully disrupted with a kanMX4 marker ${ }^{8}$.

Figure 8 Model of telomerase action during the cell cycle. Left, in $\mathrm{G} 1$ phase, a proportion of the catalytic subunit of telomerase (Est2p) is bound at the telomere in an inactive form ${ }^{18}$. In late $\mathrm{S}$ phase, the $3^{\prime}$ overhang lengthens ${ }^{12}$ and RPA, Est $1 p$ and Cdc13p become bound to the telomere ${ }^{18,19}$ (see text). Binding of Est1p depends on both $\mathrm{Cdc} 13 \mathrm{p}^{14,15,18}$ and RPA, whereas binding of RPA and Cdc13p can occur independently (see text). The complex formed between the 3' overhang, RPA, Cdc13p and Est $1 p$ is required for telomerase action at the chromosome end. The interactions between RPA, Est $1 p$, Est2p and Cdc13p are likely to be transient, indirect or both, as the complex has

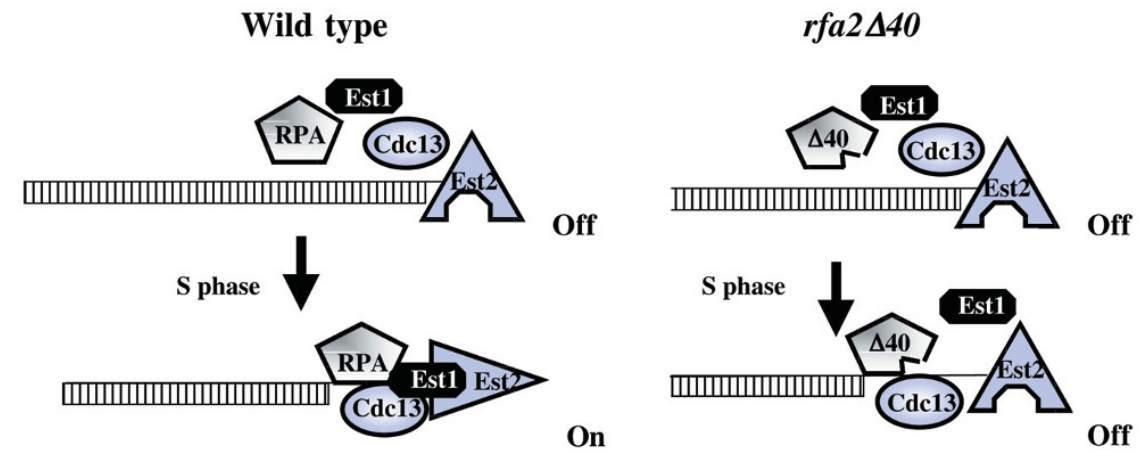
not been identified in coimmunoprecipitation experiments. Notably, in G1, we found a basal level of RPA binding to telomeres (not shown in this model). Right, in the presence of the rfa2 $\Delta 40$ mutation, the RPA complex $(\Delta 40)$ binds to the $3^{\prime}$ overhang but is unable to load Est1p. Consequently, the telomerase stays in its inactive form despite the proper fixation of $\mathrm{Cdc} 13 \mathrm{p}$. Because residual telomerase activity can be detected in $\mathrm{rfa} 2 \Delta 40$ cells, one can imagine that a weak loading of Est1p can still occur. 
Rfa2p was tagged at its C terminus with 18 MYC epitope with pGG1 (ref. 8). Epitope-tagged Cdc13p, Est1p and Est2p were constructed to retain their endogenous promoters. Cdc13p and Est2p were $18 \times$ Myc epitope-tagged in their N-terminal portions using pVL1042 and pVL1001 linearized plasmids (kindly provided by V. Lundblad). Est1p was $13 \times$ Myc epitope-tagged in its C-terminal portion using one-step PCR. The functional RFA1-MYC18 allele was obtained from T. Tanaka ${ }^{36}$. We carried out silencing assays, telomere length analysis by Southern blotting, G-strand extension at telomeres and synchronization as described ${ }^{8,16,21}$. We carried out most of the experiments in yeast strains isogenic to UCC1001 (MATa ade2-101 his34200 leu2A1 trp1A1 ura3-52 TELadh4:URA3).

We combined the rfa2 $\Delta 40$ mutation with est $2 \Delta$ as follows: we crossed the yeast strain JKM179 MAT $\alpha$ TELadh4:URA3 est2A (grown for 25 generations after the disruption of EST2) to JKM139 MATa $r f a 2 \Delta 40$ to produce the

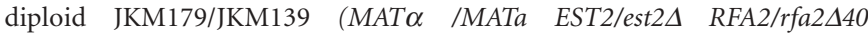

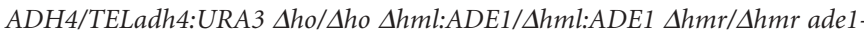
100/ade1-100 leu2-3,112/leu2-3,112 lys5/lys5 trp1:hisG/trp1:hisG ura352/ura3-52 ade3:GAL:HO/ade3:GAL:HO). Integration of a PCR product containing the nourseothricin-resistance (NAT) gene amplified from pAG25 (a kind gift from P. Goldstein and J. McCusker) disrupted EST2. We streaked the diploid strain several times in rich medium before sporulation, then identified spores with the appropriate genotypes and determined their telomere lengths as described ${ }^{16}$.

Southern blotting. For Southern blots, EcoRV- and HindIII-digested genomic DNAs were resolved in 1.2\% agarose gel and transferred to Hybond-N+ membrane (Amersham Biosciences). We probed the digested genomic DNAs with a radiolabeled URA3 DNA fragment. For Y' Southern blots, XhoI-digested genomic DNAs were resolved in $1.2 \%$ agarose gel and transferred to Hybond$\mathrm{N}+$ membrane and probed with a radiolabeled Y' DNA fragment.

Chromatin immunoprecipitations assays. We carried out ChIP assays as described $^{8}$ in yeast strains isogenic to UCC1001. Immunoprecipitation of cross-linked DNA was done with agarose-conjugated 9E10 monoclonal antibodies (to Myc; Santa Cruz Biotechnology) or with polyclonal antibodies to Rfa2p (kindly provided by S. Brill, Rutgers University, Piscataway, New Jersey and B. Stillman, Cold Spring Harbor Laboratory, Cold Spring Harbor, New York, respectively). The results obtained with anti-Rfa2p antibodies respect to the telomeric binding of Rfa2p are similar to those described obtained with the RFA2-MYC strain with anti-Myc 9E10 antibodies. We used primer pairs specific for the fragmented URA3 to amplify the 400-bp pUra2 and primer pairs specific for the telomeric $\mathrm{TG}_{1-3}$ repeats and $U R A 3$ to amplify the 230-bp pUral. The chromosome VI-R telomere-specific primers (SG355 and SG356), which have been described previously ${ }^{37}$, produced a 400 -bp fragment located $0.6 \mathrm{~kb}$ from the TG repeat. Control primers specific for the BDF1 control sequence produced a 100-bp PCR fragment. (All primer sequences are available on request.) We calculated the amounts of telomeric and nontelomeric DNAs in input and immunoprecipitated samples by quantitative PCR with a LightCycler PCR (Roche) as described ${ }^{8}$. Relative enrichment of telomeric DNA (pUra2) over background (pBdf1) in the immunoprecipitates was calculated as follows: $\left(\mathrm{IP}_{\mathrm{a}} \mathrm{pUra} / \mathrm{IP}_{\mathrm{a}} \mathrm{pBdf1}\right) \times$ (input ${ }_{a}$ pBdf1/input ${ }_{a}$ pUra2); $I_{\mathrm{a}}$ and input ${ }_{\mathrm{a}}$ represent the amount of PCR product in the immunoprecipitates and the input samples, respectively.

Yeast one-hybrid assay for telomere-interacting proteins. Yeast strains were derived from YM701 (MATa ura3-52 his3-200 ade2-101 lys2-801 trp1901 tyr 1 ). Strains HIS-Int-CA and HIS-Tel and plasmids pJG4-5/RIF2 and pJG4-5/CDC13N were kindly provided by V. Zakian ${ }^{20}$. We cloned RFA2 into pJG4-5 as an XhoI DNA fragment. To monitor telomere interaction, we assayed cells on $3 \% \mathrm{Gal}-\mathrm{His}$ test plates containing $5 \mathrm{mM} 3$-amino-1,2,4triazole (3-AT). Control plates were $3 \% \mathrm{Gal}-\mathrm{Trp}$. Plates were incubated at $30{ }^{\circ} \mathrm{C}$ for $5 \mathrm{~d}$.

\section{ACKNOWLEDGMENTS}

We thank S. Brill for antibodies to Rfa2p, V. Lundblad for providing plasmid pVL1120 and plasmids used to Myc-tag Cdc13p and Est2p, and V. Zakian for reagents for the one-hybrid assay for telomeric proteins. Work in V.G.'s laboratory was supported by l'Association pour la Recherche sur le Cancer and by la Fondation pour la Recherche, work in E.G.'s laboratory by the Ligue Nationale contre le Cancer and work in M.P.L.'s laboratory by the Associazione Italiana per la Ricerca sul Cancro and by Telethon-Italy.

\section{COMPETING INTERESTS}

The authors declare that they have no competing financial interests.

Received 30 June; accepted 1 December 2003

Published online at http://www.nature.com/naturegenetics

1. Longhese, M.P., Plevani, P. \& Lucchini, G. Replication factor A is required in vivo for DNA replication, repair, and recombination. Mol. Cell. Biol. 14, 7884-7890 (1994).

2. Wold, M.S. Replication protein A: a heterotrimeric, single-stranded DNA-binding protein required for eukaryotic DNA metabolism. Annu. Rev. Biochem. 66, 61-92 (1997).

3. Smith, J., Zou, H. \& Rothstein, R. Characterization of genetic interactions with RFA1: the role of RPA in DNA replication and telomere maintenance. Biochimie $\mathbf{8 2}, 71-78$ (2000).

4. Brill, S.J. \& Stillman, B. Replication factor-A from Saccharomyces cerevisiae is encoded by three essential genes coordinately expressed at $\mathrm{S}$ phase. Genes Dev. 5, 1589-1600 (1991)

5. Brush, G.S., Morrow, D.M., Hieter, P. \& Kelly, T.J. The ATM homologue MEC1 is required for phosphorylation of replication protein A in yeast. Proc. Natl. Acad. Sci. USA 93, 15075-15080 (1996).

6. Brush, G. S. \& Kelly, T.J. Phosphorylation of the replication protein A large subunit in the Saccharomyces cerevisiae checkpoint response. Nucleic Acids Res. 19, 3725-3732 (2000)

7. Brush, G.S., Clifford, D.M., Marinco, S.M. \& Bartrand, A.J. Replication protein A is sequentially phosphorylated during meiosis. Nucleic Acids Res. 29, 4808-4817 (2001)

8. Schramke, V. et al. The set1 $1 \Delta$ mutation unveils a novel signaling pathway relayed by the Rad53-dependent hyperphosphorylation of replication protein A that leads to transcriptional activation of repair genes. Genes Dev. 15, 1845-1858 (2001).

9. McEachern, M.J., Krauskopf, A. \& Blackburn, EH. Telomeres and their control. Annu. Rev. Genet. 34, 331-358 (2000).

10. Nugent, C.I. \& Lundblad, V. The telomerase reverse transcriptase: components and regulation. Genes Dev. 12, 1073-1085 (1998).

11. Price, C.M. Synthesis of the telomeric C-strand. A review. Biochemistry 62 , 1216-1223 (1997).

12. Wellinger, R.J., Wolf, A.J. \& Zakian, V.A. Saccharomyces telomeres acquire singlestrand $\mathrm{TG}_{1-3}$ tails late in $\mathrm{S}$ phase. Cell 72, 51-60 (1993).

13. Lin, J.J. \& Zakian, V.A. The Saccharomyces CDC13 protein is a single-strand $\mathrm{TG}_{1-3}$ telomeric DNA-binding protein in vitro that affects telomere behavior in vivo. Proc. Natl. Acad. Sci. USA 93, 13760-13765 (1996).

14. Evans, S.K. \& Lundblad, V. Est1 and Cdc13 as comediators of telomerase access. Science 286, 117-120 (1999).

15. Pennock, E., Buckley, K. \& Lundblad, V. Cdc13 delivers separate complexes to the telomere for end protection and replication. Cell 104, 387-396 (2001).

16. Marcand, S., Brevet, V. \& Gilson, E. Progressive cis-inhibition of telomerase upon telomere elongation. EMBO J. 18, 3509-3519 (1999).

17. Diede, S.J. \& Gottschling, D.E. Telomerase-mediated telomere addition in vivo requires DNA primase and DNA polymerases $\alpha$ and $\delta$. Cell 99, 723-733 (1999).

18. Taggart, A.K., Teng, S.C. \& Zakian V.A. Est1p as a cell cycle-regulated activator of telomere-bound telomerase. Science 297,1023-1026 (2002).

19. Smith, C.D., Smith, D.L., DeRisi, J.L. \& Blackburn, E.H. Telomeric protein distributions and remodeling through the cell cycle in Saccharomyces cerevisiae. Mol. Biol. Cell. 14, 556-570 (2003).

20. Bourns, B.D., Alexander, M.K., Smith, A.M. \& Zakian, V.A. Sir proteins, Rif proteins, and Cdc13p bind Saccharomyces telomeres in vivo. Mol. Cell. Biol. 18, 5600-5608 (1998).

21. Gravel, S., Larrivee, M., Labrecque, P. \& Wellinger, R.J. Yeast Ku as a regulator of chromosomal DNA end structure. Science 280, 741-744 (1998).

22. Huffman, K.E., Levene, S.D., Tesmer, V.M., Shay, J.W. \& Wright, W.E. Telomere shortening is proportional to the size of the G-rich telomeric 3'-overhang. J. Biol. Chem. 275, 19719-19722 (2000)

23. Greenwell, P.W. et al. TEL1, a gene involved in controlling telomere length in S. cerevisiae, is homologous to the human ataxia telangiectasia gene. Cell $\mathbf{8 2}, 823-829$ (1995).

24. Craven, R.J. \& Petes, T.D. Dependence of the regulation of telomere length on the type of subtelomeric repeat in the yeast Saccharomyces cerevisiae. Genetics 152, 1531-1541 (1999).

25. Peterson, S.E. et al. The function of a stem-loop in telomerase RNA is linked to the DNA repair protein Ku. Nat. Genet. 27, 64-67 (2001).

26. Polotnianka, R.M., Li, J. \& Lustig, A.J. The yeast Ku heterodimer is essential for protection of the telomere against nucleolytic and recombinational activities. Curr. Biol. 8, 831-834 (1998).

27. Dornreiter, I. et al. Interaction of DNA polymerase $\alpha$-primase with cellular replication protein A and SV40-T antigen. EMBO J. 11, 769-776 (1992).

28. Longhese, M.P., Neecke, H., Paciotti, V., Lucchini, G. \& Plevani, P. The 70 kDa subunit of replication protein $A$ is required for the G1/S and intra-S DNA damage checkpoints in budding yeast. Nucleic Acids Res. 24, 3533-3537 (1996). 


\section{ARTICLES}

29. Ritchie, K.B. \& Petes, T.D. The Mre11p/Rad50p/Xrs2p complex and the Tel1p func tion in a single pathway for telomere maintenance in yeast. Genetics 155, 475-479 (2000).

30. Chan, S.W., Chang, J., Prescott, J. \& Blackburn, E.H. Altering telomere structure allows telomerase to act in yeast lacking ATM kinases. Curr. Biol. 11, 1240-1250 (2001).

31. Tsukamoto, Y., Taggart, A.K. \& Zakian, V.A. The role of the Mre11-Rad50-Xrs2 complex in telomerase- mediated lengthening of Saccharomyces cerevisiae telomeres. Curr. Biol. 11, 1328-1335 (2001)

32. Maniar, H.S., Wilson, R. \& Brill, S. J. Roles of replication protein-A subunits 2 and 3 in DNA replication fork movement in Saccharomyces cerevisiae. Genetics 145 , 891-902 (1997)

33. Mallory, J.C. et al. Amino acid changes in Xrs2p, Dun1p, and Rfa2p that remove the preferred targets of the ATM family of protein kinases do not affect DNA repair or telomere length in Saccharomyces cerevisiae. DNA Repair 2, 1041-1064 (2003).

34. Reichenbach, P.et al. A human homolog of yeast est 1 associates with telomerase and uncaps chromosome ends when overexpressed. Curr. Biol. 13, 568-574 (2003).

35. Snow, B.E. et al. Functional conservation of the telomerase protein est1p in humans. Curr. Biol. 13, 698-704 (2003).

36. Tanaka, T. \& Nasmyth, K. Association of RPA with chromosomal replication origins requires an Mcm protein, and is regulated by Rad53, and cyclin- and Dbf4-dependent kinases. EMBO J. 17, 5182-5191 (1998).

37. Martin, S.G., Laroche, T., Suka, N., Grunstein, M. \& Gasser, S.M. Relocalization of telomeric Ku and SIR proteins in response to DNA strand breaks in yeast. Ce// $\mathbf{9 7}$ 621-633 (1999). 